\title{
An observational study of vascular endothelial growth factor inhibitors as second-line treatment for metastatic colorectal cancer treated with bevacizumab plus FOLFIRI beyond progression: the association with RAS mutation and tumor sidedness
}

\author{
Hsiang-Lin Tsai ${ }^{1,2}$, Ching-Wen Huang ${ }^{1,2}$, Cheng-Jen $\mathrm{Ma}^{1,3}$, Wei-Chih $\mathrm{Su}^{1}$, Tsung-Kun Chang ${ }^{1}$, \\ Po-Jung Chen ${ }^{1,4}$, Yung-Sung Yeh ${ }^{1,5}$, Jaw-Yuan Wang ${ }^{1,2,6,7,8}$ \\ ${ }^{1}$ Division of Colorectal Surgery, Department of Surgery, Kaohsiung Medical University Hospital, ${ }^{2}$ Department of Surgery, Faculty of Medicine, \\ College of Medicine, ${ }^{3}$ Division of General and Digestive Surgery, Department of Surgery, Kaohsiung Medical University Hospital, Kaohsiung \\ Medical University, Kaohsiung; ${ }^{4}$ Department of Surgery, Kaohsiung Municipal Hsiaokang Hospital, Kaohsiung; ${ }^{5}$ Division of Trauma and Critical \\ Care, Department of Surgery, Kaohsiung Medical University Hospital, ${ }^{6}$ Graduate Institute of Clinical Medicine, College of Medicine, ${ }^{7}$ Graduate \\ Institute of Medicine, College of Medicine, ${ }^{8}$ Center for Cancer Research, Kaohsiung Medical University, Kaohsiung \\ Contributions: (I) Conception and design: HL Tsai, JY Wang; (II) Administrative support: HL Tsai, JY Wang; (III) Provision of study materials or \\ patients: All authors; (IV) Collection and assembly of data: All authors; (V) Data analysis and interpretation: HL Tsai, JY Wang; (VI) Manuscript \\ writing: All authors; (VII) Final approval of manuscript: All authors. \\ Correspondence to: Prof. Jaw-Yuan Wang. Division of Colorectal Surgery, Department of Surgery, Kaohsiung Medical University Hospital, Kaohsiung \\ Medical University, No. 100, Tzyou 1st Road, Kaohsiung 807. Email: cy614112@ms14.hinet.net; jawyuanwang@gmail.com.
}

Background: The BRiTE and ARIES studies suggested that the continued use of bevacizumab beyond progression (BBP) was beneficial. This study investigated the efficacy and safety of the vascular endothelial growth factor inhibitors (VEGFis) bevacizumab and aflibercept as second-line treatments for patients with metastatic colorectal cancer (mCRC) that progressed following the application of bevacizumab-containing chemotherapy as a first-line treatment.

Methods: This observational cohort study (OCS) analyzed the medical records of 73 patients with mCRC divided into a no-VEGFi group $(\mathrm{n}=48)$ and a VEGFi group $(\mathrm{n}=25)$. Progression-free survival $(\mathrm{PFS})$ was the primary endpoint, and the overall survival (OS), objective response rate (ORR), disease control rate (DCR), and safety were secondary endpoints.

Results: The results revealed that the PFS, ORR, and DCR of the VEGFi group were significantly superior to those of the no-VEGFi group, even in those with wild-type and mutant-type $R A S$ or left-sided mCRC (all $\mathrm{P}<0.05$ ); however, OS did not differ significantly between the two groups (all $\mathrm{P}>0.05$ ). Patients with primary left-sided lesions and continued use of VEGFi exhibited the most marked effect on PFS $(\mathrm{P}=0.001)$. No significant differences were observed in the incidence of grade 3 or 4 adverse events (AEs) between the two groups $(\mathrm{P}=0.133)$.

Conclusions: These results support the use of VEGFi as a second-line treatment after bevacizumab beyond the initial progression in this OCS. Bevacizumab or aflibercept combined with second-line chemotherapy in mCRC has an acceptable safety profile and is relatively active. Regardless of the RAS gene type, VEGFi plus FOLOFX6 exhibited superior PFS to that of FLFOX6 as a second-line treatment, and a greater improvement in PFS was obtained for the left-sided lesions than for the right-sided lesions.

Keywords: Vascular endothelial growth factor inhibitor (VEGFis); bevacizumab beyond progression (BBP); second-line treatment; $R A S$ mutation; tumor sidedness 
Submitted Apr 25, 2019. Accepted for publication Sep 12, 2019.

doi: $10.21037 /$ tcr.2019.09.59

View this article at: http://dx.doi.org/10.21037/tcr.2019.09.59

\section{Introduction}

Curative surgical resection is a treatment for initial colorectal cancer (CRC) (1). If resection is unfeasible, then chemotherapy or target therapy is used to prolong life expectancy.

Several second-line treatment options are now available for patients for whom initial therapy had failed. The common treatment options for patients who tolerate intensive therapy include the triple drug regimen or biologic agents in combination with a chemotherapy regimen in the initial metastatic CRC (mCRC) diagnosis (2). In some phase II and III studies, bevacizumab was added to the standard 5 -fluorouracil (5-FU)-based chemotherapy regimen because it improves the survival rate compared with chemotherapy alone, as demonstrated in patients with mCRC (3-5). The bevacizumab plus FOLFOX4 regimen resulted in improved median overall survival (OS) (12.9 vs. 10.8 months) and median progression-free survival (PFS) (7.3 vs. 4.7 months) compared with the FOLFOX4 regimen (6). The ML18147 study revealed that maintenance of the vascular endothelial growth factor (VEGF) inhibitor (bevacizumab) with standard second-line chemotherapy beyond disease progression has clinical benefits for patients with mCRC (7). The RAISE study reported that adopting ramucirumab plus FOLFIRI as a second-line treatment significantly improved OS in patients with mCRC (8). In addition, the VELOUR study revealed that the addition of aflibercept to second-line FOLFIRI had benefits in patients with mCRC regardless of the timing for the first-line disease progression; no unexpected safety concerns were noted (9). However, few clinical studies have evaluated the efficacy and safety of VEGF inhibitors (VEGFi) combined with chemotherapy, which can be used as second-line therapy following firstline treatment with FOLFIRI plus bevacizumab.

The BRiTE study investigated the continued use of bevacizumab in patients with disease progression after first-line bevacizumab combination therapy (10). In the BRiTE study, the median OS in patients who were administered no bevacizumab beyond progression (BBP) was 19.9 months, whereas that of patients with BBP was 31.8 months. In agreement with the BRiTE study, another study confirmed that the continued use of bevacizumab across first- and second-line therapies prolonged OS (11). In the ARIES study, survival beyond first progression in patients administered BBP was 14.1 months, whereas that of patients who received treatment that did not include bevacizumab was 7.5 months (12). Furthermore, in the ML18147 study, the median OS was 11.2 months for patients undergoing bevacizumab plus chemotherapy and 9.8 months for those undergoing chemotherapy only (7). In the RAISE study, the median OS was 13.3 months for patients in the ramucirumab group and 11.7 months for those in the placebo group (8). In the VELOUR study, OS was higher in the aflibercept-plus-FOLFIRI arm than in the placebo-plus-FOLFIRI arm; the median OS difference was 2.14 months (9). However, these studies were subject to the limitation that the response rates were not reported.

This study assessed the efficacy and safety of VEGFi combined with standard chemotherapy regimens in patients with mCRC who exhibited disease progression after receiving bevacizumab plus FOLFIRI as a first-line therapy.

\section{Methods}

\section{Study design}

This retrospective study investigated the efficacy and safety of VEGFi as a second-line therapy in patients with mCRC that progressed after they had received bevacizumab combined with FOLFIRI as a first-line therapy. We collected the clinical outcomes of treatment, and the information obtained was from the review of actual charts and medical records. The enrolled patients were divided into two groups: a no-VEGFi group and a VEGFi group; mFOLFOX6 was selected as the second-line therapy in the no-VEGFi group, and mFOLFOX6 plus VEGFi (bevacizumab or aflibercept) was selected as the second-line therapy in the VEGFi group. The physicians determined all aspects of patients' treatments over time, including specific chemotherapy agents and combinations. VEGFis (bevacizumab or aflibercept) were not supplied by a sponsor. All clinical samples were obtained with informed consent from each patient, and the study protocol was approved by the institutional review board of Kaohsiung Medical University Hospital under approval number KMUHIRB- 
2012-03-02(II).

PFS was selected as the primary endpoint, and the secondary endpoints were OS, ORR, DCR, and safety.

\section{Patient eligibility}

Patients were considered eligible for this study if they exhibited $\mathrm{mCRC}$ with progression confirmed according to the Response Evaluation Criteria in Solid Tumors (RECIST) guidelines (13) and if they had received bevacizumab plus FOLFIRI as a first-line treatment. The time to first disease progression had to be at least 3 months. An age of $\geq 20$ years, life expectancy of $>3$ months, and Eastern Cooperative Oncology Group performance status of 0-2 were other inclusion criteria. In addition, the study patients were required to have adequate hematologic (absolute neutrophil count $>1,500 / \mu \mathrm{L}$, hemoglobin $>9.0 \mathrm{~g} / \mathrm{dL}$, and platelet count $>75,000 / \mu \mathrm{L}$ ), hepatic (bilirubin $<2.0 \mathrm{mg} / \mathrm{dL}$ and transaminase value $<3$-times the upper normal limit), and renal (creatinine $<1.5 \mathrm{mg} / \mathrm{dL}$ and urinary excretion $<500 \mathrm{mg}$ of protein per day) function.

The exclusion criteria were (I) other malignancies in the preceding 2 years; (II) presence of clinically significant cardiovascular disease, uncontrolled hypertension, or central nervous system metastasis; (III) major surgery within the preceding 6 weeks; (IV) pregnancy or lactation; nonhealing wounds; (V) bleeding diatheses; (VI) regular use of aspirin (>325 mg/day) or other nonsteroidal anti-inflammatory agents; (VII) pre-existing coagulopathies; and need for fulldose anticoagulation.

\section{Treatment}

Among the 73 patients enrolled in this study, 48 patients who received mFOLFOX6 were assigned to the noVEGFi group and 25 patients who received mFOLFOX6 plus bevacizumab or aflibercept were assigned to the VEGFi group. Right-sided colon cancer was defined as primary lesions located at the cecum, ascending colon, or transverse colon; a left-sided lesion was defined as that at the descending colon, sigmoid colon, rectosigmoid colon, or rectum. For each patient, we examined the genotyping of the $R A S$ gene.

In the no-VEGFi group, the mFOLFOX6 regimen consisted of oxaliplatin $\left(85 \mathrm{mg} / \mathrm{m}^{2}\right.$ as a 4-hour intravenous [IV] infusion) on day 1 followed by leucovorin $\left(200 \mathrm{mg} / \mathrm{m}^{2}\right.$ as a 2 -hour IV infusion) and 5 -FU $\left(2800 \mathrm{mg} / \mathrm{m}^{2}\right.$ as a 46 -hour
IV infusion); this regimen was performed biweekly. In the VEGFi group, the regimen of mFOLFOX6 plus bevacizumab or aflibercept consisted of bevacizumab ( $5 \mathrm{mg} / \mathrm{kg}$ as a 2 -hour IV infusion) or aflibercept $(4 \mathrm{mg} / \mathrm{kg}$ as a 2 -hour IV infusion) on day 1 followed by oxaliplatin (85 mg/m $\mathrm{m}^{2}$ as a 4-hour IV infusion), leucovorin $\left(200 \mathrm{mg} / \mathrm{m}^{2}\right.$ as a 2 -hour IV infusion), and 5 -FU $\left(2,800 \mathrm{mg} / \mathrm{m}^{2}\right.$ as a 46-hour IV infusion); this regimen was performed biweekly. AEs were assessed using the National Cancer Institute's Common Terminology Criteria for Adverse Events v4.02 (CTCAE v4.02) (14).

\section{Assessment}

The present study evaluated the PFS, OS, ORR, and toxicity of VEGFi in patients with $\mathrm{MCRC}$ for whom prior bevacizumab-containing treatment had failed. Failure of prior bevacizumab-containing treatment as the first-line regimen (FOLFIRI plus bevacizumab) was determined according to RECIST criteria (15). Tumor response was assessed using RECIST guidelines. Progression was defined as a $20 \%$ increase at the time of disease progression. PFS and OS were calculated using the Kaplan-Meier method. The day of introduction of second-line treatment was considered the starting point for the calculation of PFS and OS.

Toxicities were graded using the CTCAE v4.02. Radiographic assessments were performed at baseline (within 4 weeks prior to registration). For diagnostic assessment of efficacy, which was performed once every 12 weeks, computed tomography or magnetic resonance imaging was used to assess target and nontarget lesions and confirm the presence or absence of new lesions.

\section{Statistical analysis}

The means \pm standard deviation was used as continuous variables, and dichotomous variables were expressed as numbers and percentages. All statistical analyses were performed using the Statistical Package for the Social Sciences, Version 19.0 (SPSS Inc., Chicago, IL, USA). Pearson's chi-squared test was used to compare the clinicopathological characteristics of the two groups by using the Cox regression coefficients to estimate the hazard ratios for all independent variables in the model. The definitions of PFS were the time elapsed between the initiation of the study therapy and the date of disease progression, death, or the last follow-up. OS was defined as 
the time elapsed between the initiation of the study therapy and the date of death from any cause or the final follow-up. The Kaplan-Meier method was used to evaluate the PFS and OS, and the log-rank test was used to compare timeto-event distributions. $\mathrm{P}<0.05$ was considered statistically significant.

\section{Results}

\section{Patients' characteristics}

Between May 2015 and February 2018, 73 patients were enrolled in this study; all 73 were included in the efficacy and safety analysis set. The baseline characteristics of the enrolled patients are presented in Table 1. The median age was 60 years (range, 25-87 years). The median follow-up period was 11.0 months (range, 3.2-34.4 months). Overall, 61 patients $(83.6 \%)$ had left-sided mCRC and $12(16.4 \%)$ had right-sided mCRC. Moreover, 25 patients (34.2\%) exhibited mutant-type $R A S$ and 48 (65.8\%) exhibited wildtype $R A S$. Liver metastasis was more common in the noVEGFi group (47.9\%), whereas combined liver-lung metastasis was more common in the VEGFi group. The presence of multiple metastatic sites was more common in the VEGFi group than in the no-VEGFi group (48.0 vs. $27.1 \% ; \mathrm{P}=0.074)$.

\section{Efficacy for all 73 enrolled patients with $m C R C$}

During the median follow-up of 11.0 months (range, 3.234.4 months), 13 patients (27.1\%) and 18 patients (72.0\%) achieved disease control in the no-VEGFi and VEGFi groups, respectively $(\mathrm{P}<0.001$, Table 1$)$. Five patients had partial responses, resulting in ORRs of $20 \%$ in the VEGFi group and $0 \%$ in the no-VEGFi group $(\mathrm{P}=0.001$, Table 1$)$. The median PFS was 9.5 and 3.5 months in the VEGFi and no-VEGFi groups, respectively $[\mathrm{P}<0.001$; HR: 0.320; 95\% confidence interval (CI): $0.174-0.589$; Figure $1 A]$. The median OS was 15.5 and 10.5 months in the VEGFi and no-VEGFi groups, respectively ( $\mathrm{P}=0.083$; HR: $0.589 ; 95 \%$ CI: 0.320-1.085; Figure 1B).

\section{Safety of all 73 enrolled patients with $m C R C$}

In the no-VEGFi group, 10 patients (20.8\%) developed grade 3 or 4 toxicities, namely neutropenia in three patients, diarrhea in two patients, and oral mucositis in five patients.
In the VEGFi group, 6 patients (24.0\%) developed grade 3 or 4 toxicities, namely neutropenia in two patients, diarrhea in one patient, proteinuria in one patient, and liver function impairment in two patients. A nonsignificant difference was observed in the incidence of severe AEs between the two groups $(\mathrm{P}=0.133$; Table 1).

\section{Efficacy from the perspective of the RAS gene}

In total, 48 patients (65.8\%) had wild-type $R A S$ and 25 patients $(34.2 \%)$ had mutant-type $R A S$. Regardless of whether the $R A S$ gene was wild-type or mutant-type (Table 2), the ORR of the VEGFi group was superior to that of the no-VEGFi group ( $\mathrm{P}=0.011$ and 0.049 , respectively), and the DCR of the VEGFi group was superior to that of the no-VEGFi group ( $\mathrm{P}=0.004$ and 0.017 , respectively). In the VEGFi group, of the 16 patients with wild-type $R A S$ mCRC, $3(18.8 \%)$ and $12(75.0 \%)$ achieved ORRs and DCRs, respectively. In comparison, of the nine patients with mutant-type RAS mCRC, 2 (22.3\%) and 6 (66.7\%) achieved ORRs and DCRs, respectively (Table 2). The effects of the RAS genotype on ORRs and DCRs did not differ significantly in the VEGFi group (ORR: $\mathrm{P}=0.835$; DCR: $\mathrm{P}=0.656$ ).

For the 48 patients with wild-type $R A S$ mCRC, median PFS was 9.5 and 3.5 months in the VEGFi and noVEGFi groups, respectively ( $\mathrm{P}=0.003$; HR: 0.345 ; $95 \%$ CI: 0.162-0.736; Figure 2A); the median OS did not differ significantly between the two groups (15.5 vs. 13.5 months; $\mathrm{P}=0.137$; HR: 0.567; 95\% CI: 0.264-1.217; Figure 2B). For the 25 patients with mutant-type $R A S$ mCRC, the median PFS was 12.0 and 3.5 months and median OS was 10.0 and 8.3 months in the VEGFi and no-VEGFi groups, respectively. Thus, PFS differed significantly between the two groups ( $\mathrm{P}=0.006$; HR: 0.245 ; 95\% CI: $0.080-0.753$; Figure $3 A$ ) but $\mathrm{OS} \operatorname{did}$ not $(\mathrm{P}=0.631$; HR: 0.745 ; $95 \% \mathrm{CI}$ : 0.231-2.400; Figure 3B).

\section{Efficacy according to primary lesion location}

Of the patients with mCRC, $61(83.6 \%)$ had primary leftsided colon lesions; the other patients (16.4\%) had primary right-sided colon lesions. The ORRs and DCRs in patients with left-sided mCRC in the VEGFi group were superior to those of the corresponding patients in the no-VEGFi group $(\mathrm{P}=0.011$ and $\mathrm{P}<0.001$, respectively; Table 3). However, the ORRs and DCRs did not differ significantly between 
Table 1 Baseline clinical characteristics of $73 \mathrm{mCRC}$ patients undergoing second-line treatment

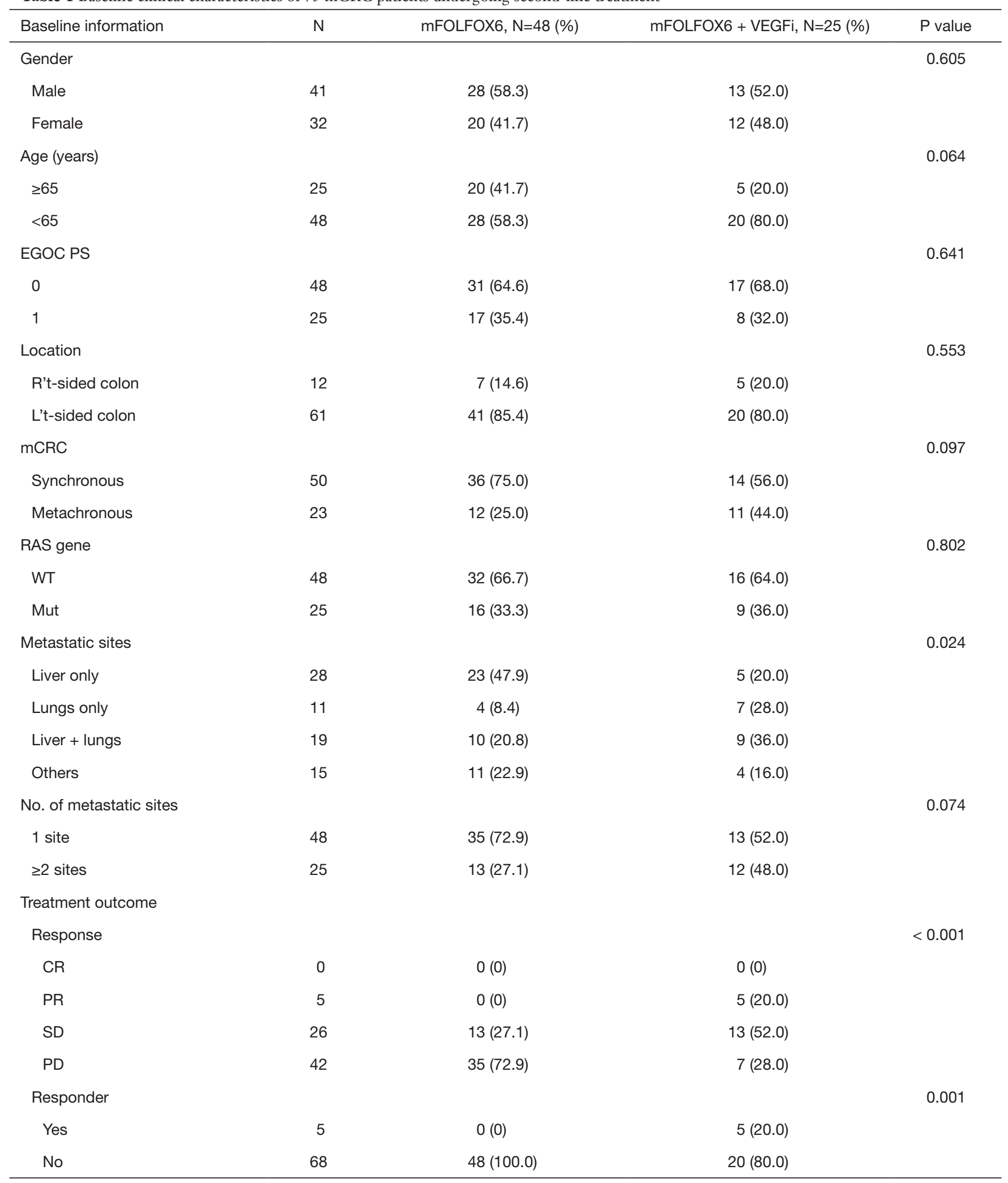

Table 1 (continued) 
Table 1 (continued)

\begin{tabular}{lccc}
\hline Baseline information & $\mathrm{N}$ & mFOLFOX6, N=48 (\%) & mFOLFOX6 + VEGFi, N=25 (\%) \\
\hline Disease control rate & 31 & $13(27.1)$ & $18(72.0)$ \\
Yes & 42 & $35(72.9)$ & $7(28.0)$ \\
No & 16 & $10(20.8)$ & $6(24.0)$ \\
Grade 3/4 AE & & 3 & 2 \\
Total & & 2 & 1 \\
Neutropenia & 5 & 0.133 \\
Diarrhea & 0 & 1 \\
Mucositis & 0 & 2 \\
Proteinuria & & 001
\end{tabular}

mCRC, metastatic colorectal cancer; VEGFi, vascular endothelial growth factor inhibitors (aflibercept and bevacizumab); R't-sided colon, cecum + ascending colon + transverse colon; L't-sided colon, descending colon + sigmoid colon + rectosigmoid colon + rectum; Wt: wild type; Mut, mutation type; others, included peritoneal seeding, para-aortic lymph nodes, neck lymph nodes; No. of metastatic sites, number of metastatic sites; response, best response during treatment; CR, complete response; PR, partial response; SD, stable disease; $\mathrm{PD}$, progressive disease; responder, $\mathrm{CR}+\mathrm{PR}$; $\mathrm{DCR}, \mathrm{CR}+\mathrm{PR}+\mathrm{SD} ; \mathrm{AE}$, adverse events.

A

$\begin{array}{lcc} & \text { No. of patients } & \text { Median PFS (ms) } \\ \text { mFOLFOX6 only } & 48 & 3.5 \\ \text { mFOLFOX6 + VEGFi } & 25 & 9.5\end{array}$

HR, 0.320; $95 \% \mathrm{Cl}, 0.174-0.589$

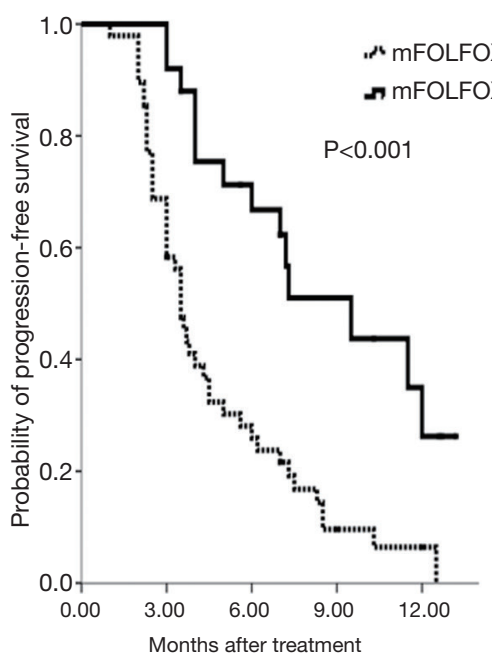

No. at risk

$\begin{array}{lrrrrr}\text { mFOLFOX6 only } & 48 & 33 & 13 & 4 & 2 \\ \text { mFOLFOX6 + VEGFi } 25 & 25 & 16 & 8 & 4\end{array}$
B

$\begin{array}{lll}\text { mFOLFOX6 only } & 48 & 10.5 \\ \text { mFOLFOX6 + VEGFi } & 25 & 15.5\end{array}$

$\mathrm{HR}, 0.589 ; 95 \% \mathrm{Cl}, 0.320-1.085$
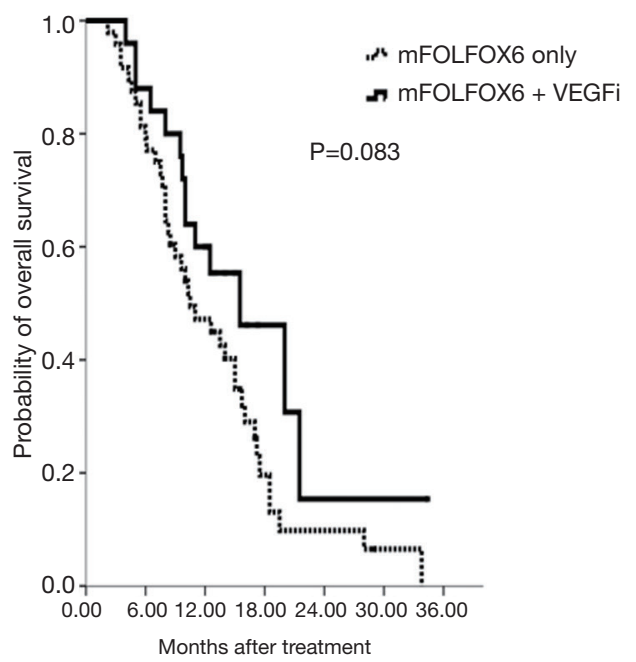

No. at risk

mFOLFOX6 only $\quad 48 \quad 39 \quad 21 \quad 6 \quad 3 \quad 3 \quad 1$

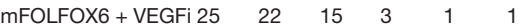

Figure 1 Cumulative survival rates of the 73 enrolled patients with mCRC, obtained using the Kaplan-Meier method. The difference in survival rates was analyzed using the log-rank test. (A) PFS was significantly longer in the VEGFi group than in the no-VEGFi group (9.5 vs. 3.5 months; $\mathrm{P}<0.001$ ); (B) OS did not differ significantly between the two groups (15.5 vs. 10.5 months; $\mathrm{P}=0.083$ ). mCRC, metastatic colorectal cancer; PFS, progression-free survival; VEGFi, vascular endothelial growth factor inhibitor; OS, overall survival. 
Table 2 Comparison of ORRs and DCRs between $48 \mathrm{mCRC}$ patients with wild-type $R A S$ and $25 \mathrm{mCRC}$ patients with mutant-type $R A S$ in the two groups of second-line treatment

\begin{tabular}{|c|c|c|c|c|c|c|}
\hline Variable & \multicolumn{3}{|c|}{ Wild type of RAS gene } & \multicolumn{3}{|c|}{ Mutant type of RAS gene } \\
\hline Response & & & 0.003 & & & 0.030 \\
\hline PR & $0(0)$ & $3(18.8)$ & & $0(0)$ & $2(22.3)$ & \\
\hline Responder & & & 0.011 & & & 0.049 \\
\hline Yes & $0(0)$ & $3(18.8)$ & & $0(0)$ & $2(22.3)$ & \\
\hline No & $32(100.0)$ & $13(81.2)$ & & $16(100.0)$ & $7(77.7)$ & \\
\hline
\end{tabular}

ORR, objective response rate; DCR, disease control rate; mCRC, metastatic colorectal cancer; VEGFi, vascular endothelial growth factor inhibitors (aflibercept and bevacizumab); PR, partial response; SD, stable disease; PD, progressive disease. Responder: yes, partial response; no, stable disease + progressive disease.

A

$\begin{array}{lcc} & \text { No. of patients } & \text { Median PFS (ms) } \\ \text { mFOLFOX6 only } & 32 & 3.5 \\ \text { mFOLFOX6+VEGFi } & 16 & 9.5\end{array}$

$\mathrm{HR}, 0.345 ; 95 \% \mathrm{Cl}, 0.162-0.736$

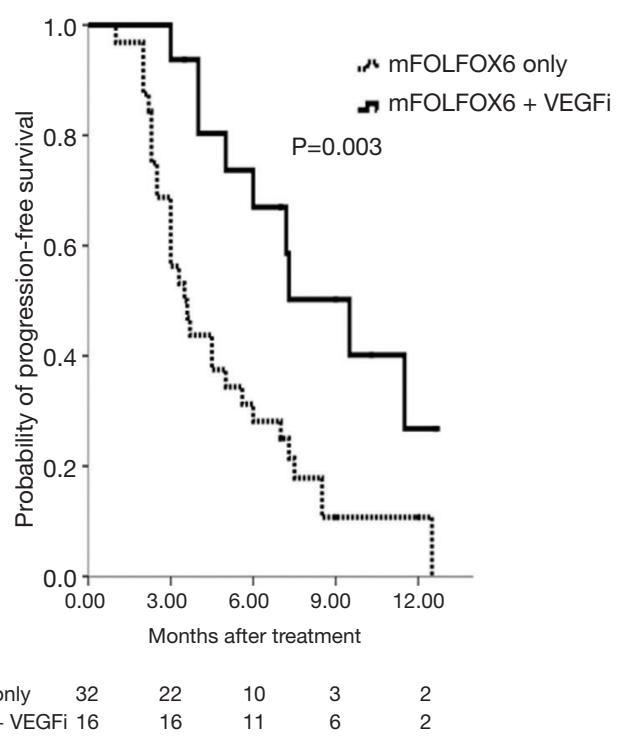

B

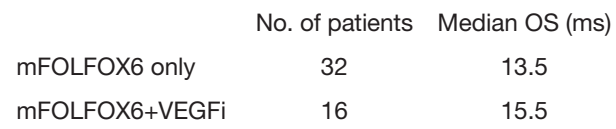

$\mathrm{HR}, 0.567 ; 95 \% \mathrm{Cl}, 0.264-1.217$

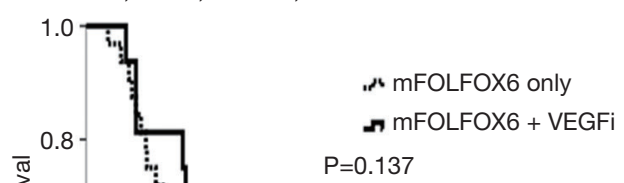

No. at risk $\begin{array}{lrrrrrr}\text { mFOLFOX6 only } & 32 & 26 & 16 & 5 & 2 & 1 \\ \text { mFOLFOX6 + VEGFi } 16 & 13 & 10 & 3 & 1 & 1\end{array}$

Figure 2 Cumulative survival rates of the 48 enrolled patients with wild-type $R A S \mathrm{mCRC}$, obtained using the Kaplan-Meier method. The difference in survival rates was analyzed using the log-rank test. (A) PFS was significantly longer in the VEGFi group than in the no-VEGFi group (9.5 vs. 3.5 months; $\mathrm{P}=0.003$ ); (B) OS did not differ significantly between the two groups (15.5 vs. 13.5 months; $\mathrm{P}=0.137)$. mCRC, metastatic colorectal cancer; PFS, progression-free survival; VEGFi, vascular endothelial growth factor inhibitor; OS, overall survival. 
A

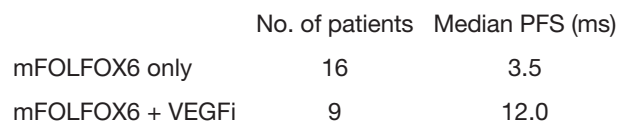

$\mathrm{HR}, 0.245 ; 95 \% \mathrm{Cl}, 0.080-0.753$

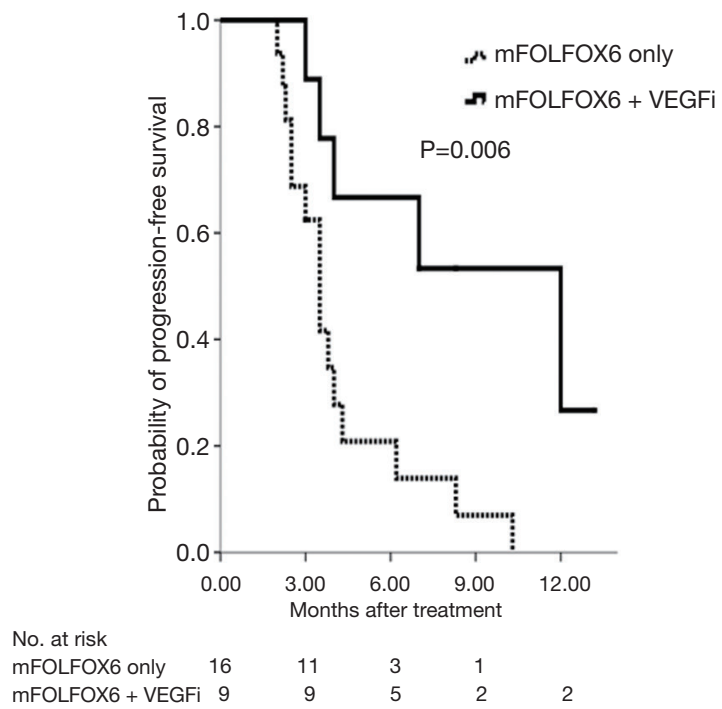

B

$\begin{array}{lcc} & \text { No. of patients } & \text { Median OS (ms) } \\ \text { mFOLFOX6 only } & 16 & 8.3 \\ \text { mFOLFOX6 + VEGFi } & 9 & 10.0\end{array}$

$\mathrm{HR}, 0.745 ; 95 \% \mathrm{Cl}, 0.231-2.400$

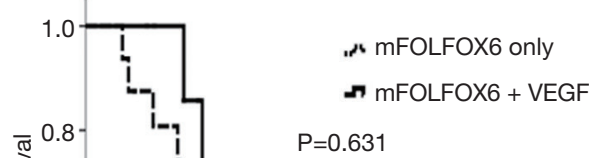

No. at risk

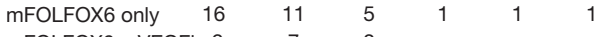

Figure 3 Cumulative survival rates of the 25 enrolled patients who had mCRC with mutant-type $R A S$, obtained using the Kaplan-Meier method. The difference in survival rates was analyzed using the log-rank test. (A) PFS was significant longer in the VEGFi group than in the no-VEGFi group (12.0 vs. 3.5 months; $\mathrm{P}=0.006$ ); (B) OS did not differ significantly between the two groups (10.0 vs. 8.3 months; $\mathrm{P}=0.631$ ). mCRC, metastatic colorectal cancer; PFS, progression-free survival; VEGFi, vascular endothelial growth factor inhibitor; OS, overall survival.

Table 3 Comparison of ORRs and DCRs between 61 Left-Sided mCRC patients and 12 right-sided mCRC patients in the two groups of secondline treatment

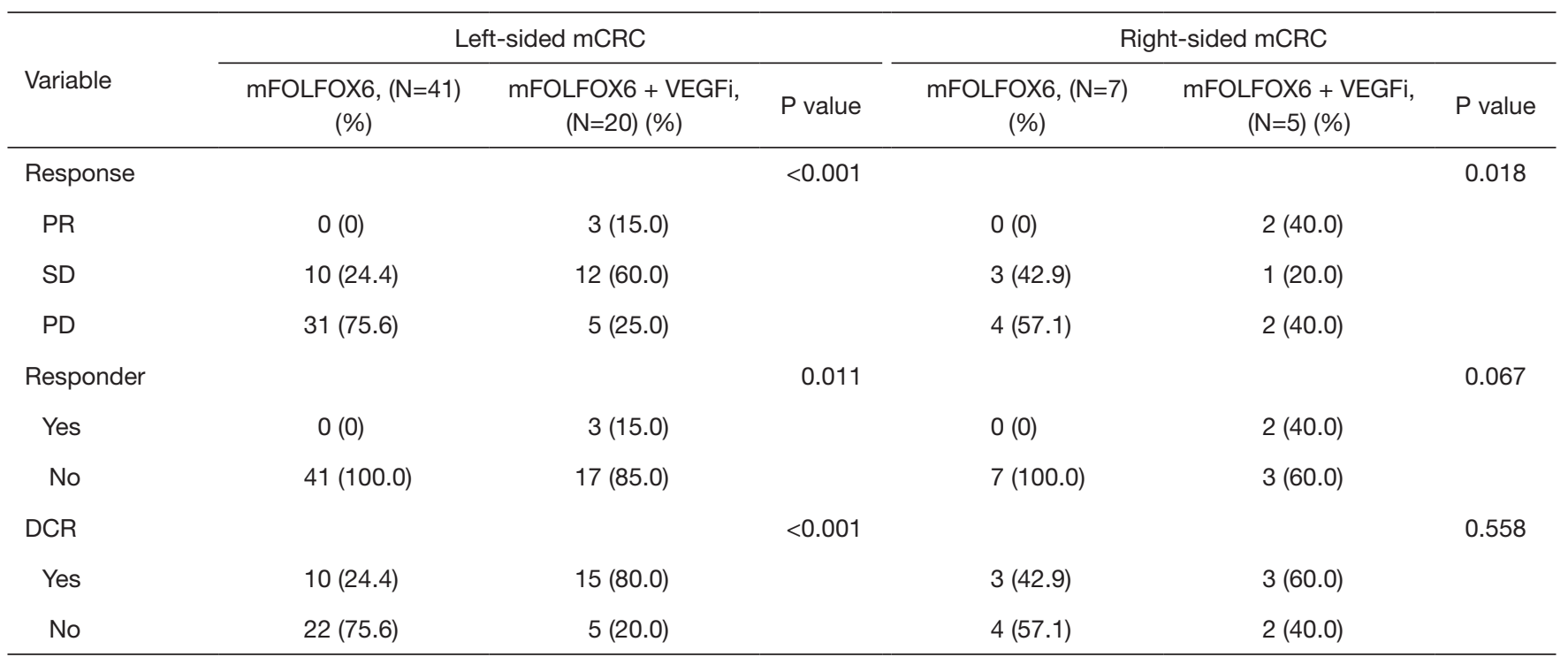

ORR, objective response rate; DCR, disease control rate; $\mathrm{mCRC}$, metastatic colorectal cancer; VEGFi, vascular endothelial growth factor inhibitors (aflibercept and bevacizumab); PR, partial response; SD, stable disease; PD, progressive disease. Responder: yes, partial response; no, stable disease + progressive disease. 
A

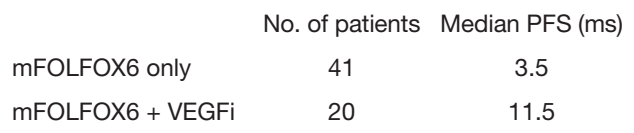

$\mathrm{HR}, 0.263 ; 95 \% \mathrm{Cl}, 0.130-0.532$

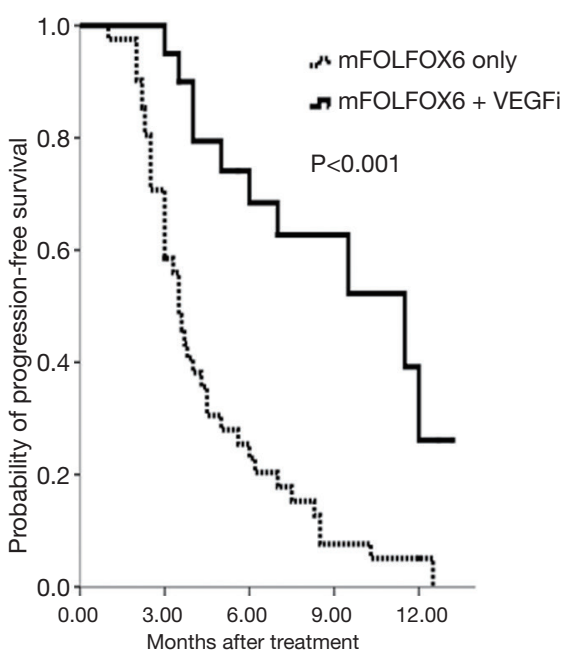

B

$\begin{array}{lcc} & \text { No. of patients } & \text { Median OS (ms) } \\ \text { mFOLFOX6 only } & 41 & 11.0 \\ \text { mFOLFOX6 + VEGFi } & 20 & 20.0\end{array}$

$\mathrm{HR}, 0.497 ; 95 \% \mathrm{Cl}, 0.245-1.008$
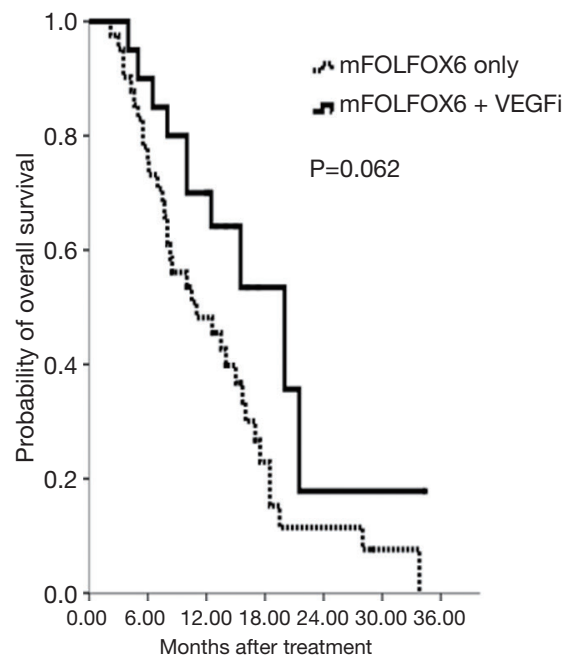

No. at risk

mFOLFOX6 only $\quad \begin{array}{llllll}48 & 32 & 18 & 6 & 3 & 1\end{array}$

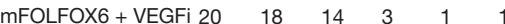

Figure 4 Cumulative survival rates of the 61 enrolled patients with left-sided mCRC, obtained using the Kaplan-Meier method. The difference in survival rates was analyzed using the log-rank test. (A) PFS was significantly longer in the VEGFi group than in the no-VEGFi group (11.5 vs. 3.5 months; $\mathrm{P}<0.001)$; (B) OS did not differ significantly between the two groups (20.0 vs. 11.0 months; $\mathrm{P}=0.062)$. mCRC, metastatic colorectal cancer; PFS, progression-free survival; VEGFi, vascular endothelial growth factor inhibitor; OS, overall survival.

the two groups among patients with right-sided mCRC $(\mathrm{P}=0.067$ and 0.558 , respectively; Table 3$)$.

The median PFS was 11.5 months in patients with leftsided $\mathrm{mCRC}$ and 3.5 months in patients with right-sided mCRC; the difference was significant $(\mathrm{P}<0.001$; HR: 0.263; 95\% CI: 0.130-0.532; Figure 4A). However, no significant difference was observed in median OS between the two groups (20.0 vs. 11.0 months; $\mathrm{P}=0.062$; HR: 0.497; 95\% CI: 0.245-1.008; Figure 4B). Nonsignificant differences were observed in median PFS (7.2 vs. 3.5 months; $\mathrm{P}=0.696$; HR: 0.946; 95\% CI: 0.250-3.587; Figure $5 A$ ) and median OS (9.7 vs. 10.3 months; $\mathrm{P}=0.543$; HR: 1.534 ; 95\% CI: 0.382-6.154; Figure $5 B$ ) in patients with right-sided $\mathrm{mCRC}$.

The 73 patients were further divided into four subgroups according to the primary mCRC location and whether continued use of VEGFi was applied in the second-line therapy. The results indicated that patients with primary left-sided lesions and continued use of VEGFi exhibited the most marked effect in PFS compared with patients in the other three subgroups ( $\mathrm{P}=0.001$; Figure $)$.

\section{Discussion}

The present study evaluated treatment patterns and clinical outcomes in patients with mCRC who had been administered FOLFIRI and bevacizumab as a first-line treatment. We demonstrated that the continued use of VEGFi outperformed mFOLFOX6 alone as a second-line regimen in terms of its effects on PFS, ORR, and DCR in patients with the wild-type or mutant-type $R A S$ gene or with primary left-sided mCRC. This study also revealed that the toxicities were not significantly different between the two groups. To date, few randomized clinical studies have evaluated the effect of BBP continuation in patients with mCRC for the purpose of examining the clinical benefits of sustained VEGF suppression.

Disease progression generally represents resistance to therapy and guides changes in therapy regimens. The genetic instability inherent in cancer renders mutant cells insensitive to primary and secondary cytotoxic drugs and results in resistance. The appearance of tumor cells that are resistant to cytotoxic regimens does not 
A

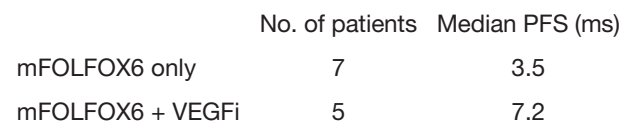

$\mathrm{HR}, 0.946 ; 95 \% \mathrm{Cl}, 0.250-3.587$

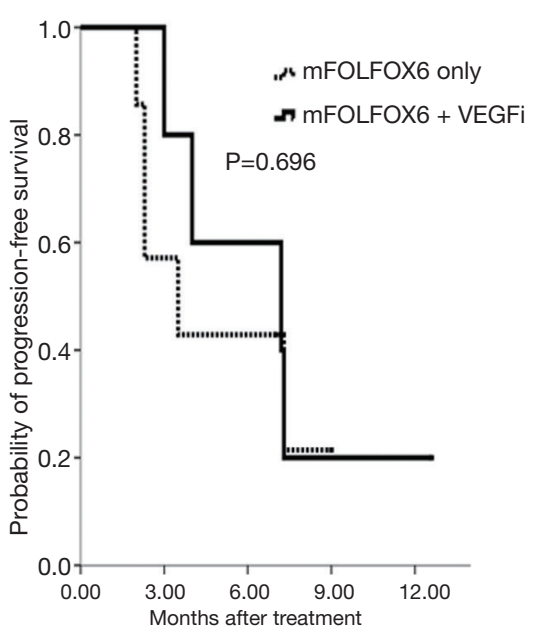

B

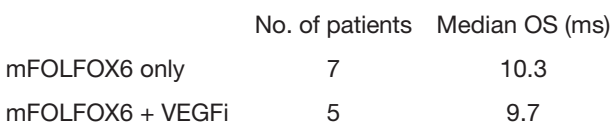

$\mathrm{HR}, 1.534 ; 95 \% \mathrm{Cl}, 0.382-6.154$

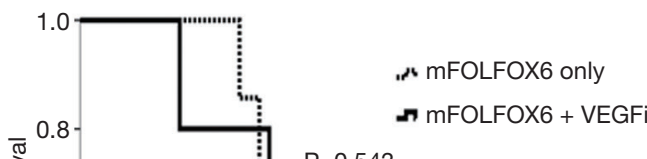

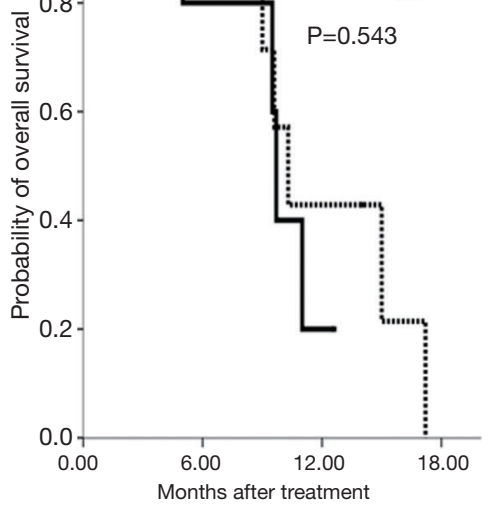

No. at risk $\begin{array}{llllll}\text { mFOLFOX6 only } & 7 & 4 & 3 & 1 & \\ \text { mFOLFOX6 + VEGFi } & 5 & 5 & 3 & 1 & 1\end{array}$

No. at risk mFOLFOX6 only $\begin{array}{llll}7 & 7 & 3\end{array}$ mFOLFOX6 + VEGFi $5 \quad 4$

Figure 5 Cumulative survival rates of the 12 enrolled patients with right-sided mCRC, obtained using the Kaplan-Meier method. The difference in survival rates was analyzed using the log-rank test. (A) PFS did not differ significantly between the two groups (7.2 vs. 3.5 months; $\mathrm{P}=0.696$ ); (B) OS was also not significantly different the two groups (9.7 vs. 10.3 months; $\mathrm{P}=0.543$ ). mCRC, metastatic colorectal cancer; PFS, progression-free survival; OS, overall survival.

definitively indicate that the disease is no longer partially or significantly dependent on VEGF-mediated endothelial cell mitogenesis and survival. One theory is that sustained VEGF suppression alongside secondary and tertiary cytotoxic regimens may result in sustained clinical benefits. The results of BBP survival analyses in the BRiTE (10) and ARIES (11) studies provide powerful evidence to support this hypothesis.

VEGF is the most influential mediator of angiogenesis and the sole angiogenic factor that has been shown to be expressed not only in the initial stages of tumor growth but also throughout the entire tumor life cycle (16). The development of resistance to bevacizumab is believed to be attributable to the apparent attenuation of its effects on other angiogenic factors, such as basic fibroblast growth factor and platelet-derived growth factor, and not to the attenuation of its effects on VEGF; this suggests that the continued use of VEGFi has partial or supplemental efficacy (17). In 2012, Tsutsumi et al. reported results that provide further evidence to support this assertion (18).

The present study showed that the VEGFi group treatment was active, with an ORR of $20 \%$ and overall DCR of $72 \%$. Response and survival data in the present study seem more favorable than those reported in other studies. Efficacy data reported in other phase II studies vary considerably, with most studies reporting a median PFS of $\leq 5$ months and median OS of $<13$ months in second-line treatment settings (19-22). According to our data, the PFS was 9.5 months and the OS was 15.5 months in the VEGFi group. Notably, the PFS and OS values observed in the present study are similar to those reported in E3200 (6).

The effects of long-term exposure to VEGFis in patients who have received BBP are a concern. The results of the BRiTE study indicated no significant increase in serious AEs (SAEs) in the BBP group compared with those in the no-BBP group; these findings are similar to those of the present study. As described in previous reports $(3,6)$, SAEs are believed to be controllable through similar management approaches. Regarding safety, the incidence of SAEs due to the long-term use of VEGFi was not higher in the noVEGFi group. The observation of selected SAEs (i.e., any SAE assumed to be related to VEGFi, including 


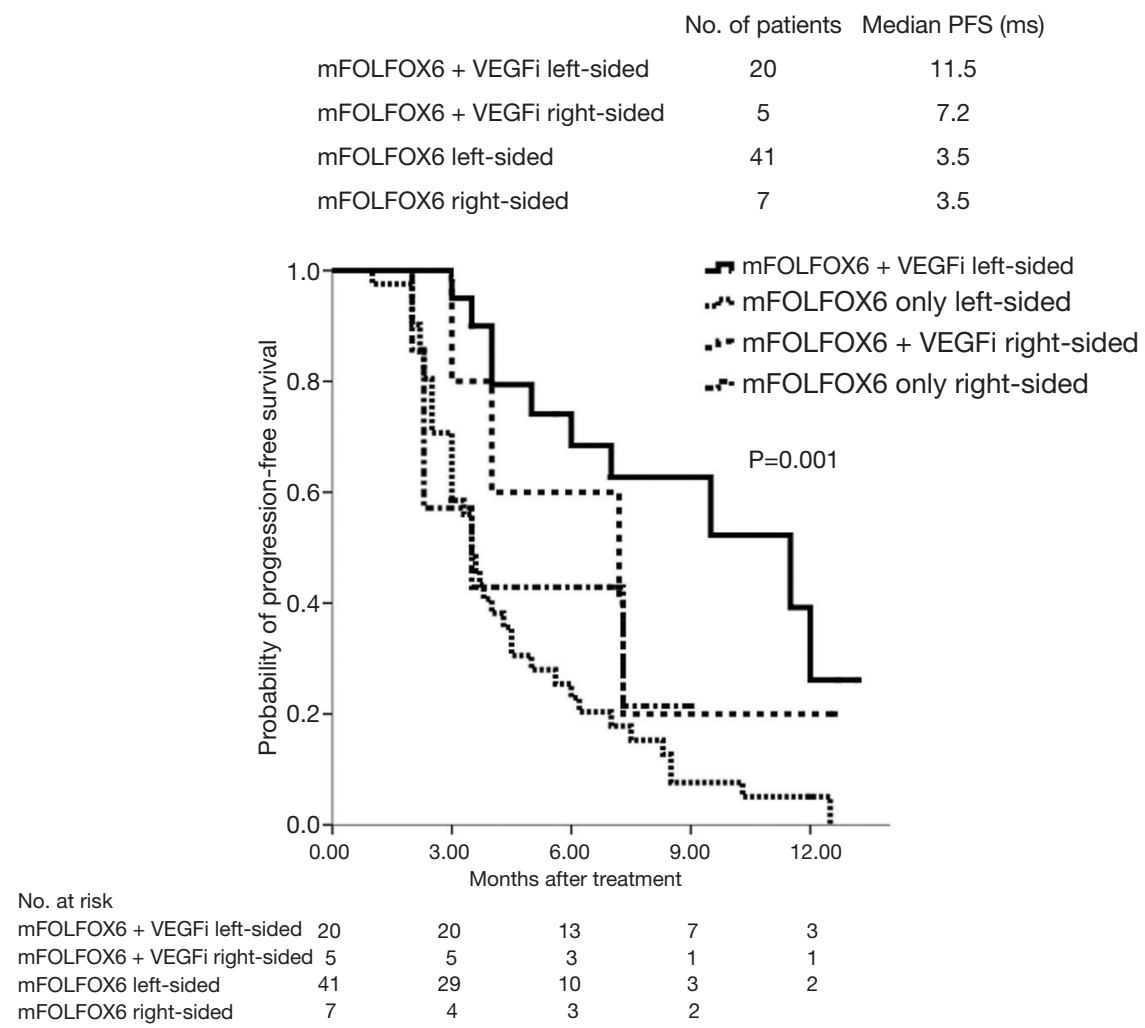

Figure 6 Cumulative survival rates of the four subgroups of patients with mCRC, obtained using the Kaplan-Meier method. The difference in survival rates was analyzed using the log-rank test. PFS in the left-sided mCRC with VEGFi subgroup was statistically superior to those in the other three subgroups $(\mathrm{P}=0.001)$. mCRC, metastatic colorectal cancer; PFS, progression-free survival; VEGFi, vascular endothelial growth factor inhibitor.

gastrointestinal perforation, arterial thromboembolic events, bleeding, and hypertension) revealed no such difference between the two groups in the present study. However, the higher cumulative incidence of grade 1 or 2 hypertension in the VEGFi group was expected given that the risk of developing bevacizumab-associated hypertension appeared to be constant over time (23) and that the VEGFi group had substantially longer bevacizumab exposure. Our study obtained similar outcomes for the cumulative incidence of grade 1 or 2 hypertension, namely $23.5 \%$ and $18.3 \%$ in the VEGFi and no-VEGFi groups, respectively.

The European Prospective Investigation into Cancer and Nutrition (EPIC) study and study 181 serve as examples of phase III clinical studies assessing second-line therapy. In the EPIC study (24), the response rate for cetuximab plus irinotecan was $16.4 \%$, and in study 181 , the response rates for panitumumab plus FOLFIRI were $35 \%$ in patients with wild-type $R A S$ and $13 \%$ in patients with mutanttype $R A S$ (19). In the present study, the response rate for mFOLFOX6 plus VEGFi as a second-line regimen was $18.8 \%$ in patients with wild-type $R A S$ and $22.3 \%$ in patients with mutant-type $R A S$. The response rates for mFOLFOX6 plus VEGFi in this study, which applied BBP, are comparable to those in patients with mutant-type $R A S$ in study 181 .

In 1990, Bufill described CRC according to primary tumor locations (25). Different origins lead to tumors with different gene expression and mutation profiles. In particular, right-sided tumors exhibit a higher frequency of $B R A F$ mutation and microsatellite instability and occur more often in patients with a genetic predisposition to CRC (e.g., Lynch syndrome). By contrast, left-sided tumors are characterized by chromosomal instability and a gene expression profile that involves activation of the epidermal growth factor receptor pathway $(26,27)$. These differences result in different prognoses for the two tumor types, and right-sided tumors are associated with poorer clinical outcomes (26-28). In another study, patients with 
right-sided mCRC who underwent second-line treatments exhibited low ORRs, especially those in the FOLFIRI arm (29). In this study, left-sided patients with mCRC and BBP who had continued use of VEGFi as a second-line treatment exhibited favorable ORRs and DCRs; patients with right-sided mCRC experienced no such benefits. Furthermore, the 73 patients enrolled were divided into four subgroups according to primary tumor location and VEGFi administration mode; the left-sided VEGFi group had significantly longer PFS than did the other subgroups.

Several limitations of this study must be considered. All OCSs have inherent limitations resulting largely from the nonrandom assignment of patients to the treatment groups being compared. Second, only 25 patients with mCRC were included in the VEGFi group in this study. In a subgroup analysis with a small number of patients, no statistical significance is not meaningful. Third, the median follow-up duration was only 11.0 months. Nevertheless, this real-world study provides insights into the effects of VEGFi combined with a range of chemotherapy regimens commonly used in patients who experience disease progression after first-line therapy.

\section{Conclusions}

In conclusion, this study demonstrates a survival benefit associated with the continuation of VEGFi treatment beyond disease progression in patients with mCRC who have received bevacizumab-containing chemotherapy as a first-line therapy. The results support the hypothesis that continued suppression of the VEGF pathway may maximize the clinical benefits of bevacizumab in patients with $\mathrm{mCRC}$, especially patients with left-sided mCRC, irrespective of $R A S$ mutation.

\section{Acknowledgments}

Funding: This work was supported by grants through funding from the Ministry of Science and Technology (MOST108-2321-B-037-001, MOST107-2321-B-037-003, MOST107-2314-B-037-116, MOST107-2314-B-037-022MY2, MOST107-2314-B-037-023-MY2) and the Ministry of Health and Welfare (MOHW107-TDU-B-212-123006, MOHW107-TDU-B-212-114026B, MOHW108TDU-B-212-133006, MOHW108-TDU-B-212-124026) funded by Health and Welfare Surcharge of Tobacco Products, and the Kaohsiung Medical University Hospital (KMUH107-7R28, KMUH107-7R29, KMUH107-7R30,
KMUH107-7M22, KMUH107-7M23, KMUHS10701, KMUHS10801, KMUHS10804, KMUHS10807) and Center for Cancer Research, Kaohsiung Medical University (KMU-TC108A04). In addition, this study was supported by the Grant of Taiwan Precision Medicine Initiative and Biomarker Discovery in Major Diseases of Taiwan Project (AS-BD-108-1), Academia Sinica.

\section{Footnote}

Conflicts of Interest: The authors have completed the ICMJE uniform disclosure form (available at http://dx.doi. org/10.21037/tcr.2019.09.59). The authors have no conflicts of interest to declare.

Ethical Statement: The authors are accountable in all respects for ensuring that questions related to the accuracy or integrity of any part of the work are appropriately investigated and resolved. The study was conducted in accordance with the Declaration of Helsinki (as revised in 2013). All clinical samples were obtained with informed consent from each patient, and the study protocol was approved by the institutional review board of Kaohsiung Medical University Hospital under approval number KMUHIRB-2012-03-02(II).

Open Access Statement: This is an Open Access article distributed in accordance with the Creative Commons Attribution-NonCommercial-NoDerivs 4.0 International License (CC BY-NC-ND 4.0), which permits the noncommercial replication and distribution of the article with the strict proviso that no changes or edits are made and the original work is properly cited (including links to both the formal publication through the relevant DOI and the license). See: https://creativecommons.org/licenses/by-nc-nd/4.0/.

\section{References}

1. Tsai HL, Yang IP, Lin CH, et al. Predictive value of vascular endothelial growth factor overexpression in early relapse of colorectal cancer patients after curative resection. Int J Colorectal Dis 2013;28:415-24.

2. National Comprehensive Cancer Network. Clinical Practice Guidelines in Oncology v2 2018. Available online: http://www.nccn.org/professionals/physician_gls/PDF/ colon.pdf. Accessed June 2018.

3. Hurwitz H, Fehrenbacher L, Novotny W, et al. Bevacizumb plus irinotecan, fluorouracil, and leucovorin 
for metastatic colorectal cancer. N Engl J Med 2004;350:2335-42.

4. Kabbinavar F, Hurwitz HI, Fehrenbacher L, et al. Phase II, randomized trial comparing bevacizumab plus fluorouracil (FU)/leucovorin (LV) with FU/LV alone in patients with metastatic colorectal cancer. J Clin Oncol 2003;21:60-5.

5. Saltz LB, Clarke S, Diaz-Rubio E, et al. Bevacizumab in combination with oxaliplatin-based chemotherapy as firstline therapy in metastatic colorectal cancer: a randomized phase III study. J Clin Oncol 2008;26:2013-9.

6. Giantonio BJ, Catalano PJ, Meropol NJ, et al.

Bevacizumab in combination with oxaliplatin, fluorouracil, and leucovorin (FOLFOX4) for previously treated metastatic colorectal cancer: results from the Eastern Cooperative Oncology Group Study E3200. J Clin Oncol 2007;25:1539-44.

7. Bennouna J, Sastre J, Arnold D, et al. Continued of bevacizumab after progression in metastatic colorectal cancer (ML 18147): a randomized phase 3 trial. Lancet Oncol 2013;14:29-37.

8. Tabernero J, Yoshino T, Cohn AL, et al. Ramucirumab versus placebo in combination with second-line FOLFIRI in patients with metastatic colorectal carcinoma that progressed during or after first-line therapy with bevacizumab, oxaliplatin, and a fluoropyrimidine (RAISE): a randomized, double-blind, multicenter, phase 3 study. Lancet Oncol 2015;16:499-508.

9. Van Cutsem E, Joulain F, Hoff PM, et al. Aflibercept Plus FOLFIRI vs. Placebo Plus FOLFIRI in Second-Line Metastatic Colorectal Cancer: a Post Hoc Analysis of Survival from the Phase III VELOUR Study Subsequent to Exclusion of Patients who had Recurrence During or Within 6 Months of Completing Adjuvant OxaliplatinBased Therapy. Target Oncol 2016;11:383-400.

10. Grothey A, Sugrue MM, Purdie DM, et al. Bevacizumab beyond first progression is associated with prolonged overall survival in metastatic colorectal cancer: Results from a large observational cohort study (BRiTE). J Clin Oncol 2008;26:5326-34.

11. Cohn AL, Bekaii-Saab T, Bendell JC, et al. Clinical outcomes in bevacizumab-treated patients with metastatic colorectal cancer: results from ARIES observational cohort study and confirmation of BRiTE data on bevacizumab beyond progression (BBP). J Clin Oncol 2010;28:abstr 3596.

12. Bendell JC, Bekaii-Saab TS, Cohn AL, et al. Treatment patterns and clinical outcomes in patients with metastatic colorectal cancer initially treated with FOLFOX- bevacizumab or FOLFIRI-bevacizumab: results from ARIES, a bevacizumab observational cohort study. Oncologist 2012;17:1486-95.

13. Eisenhauer EA, Therase P, Bogaerts J, et al. New response evaluation criteria in solid tumours: revised RECIST guideline (version 1.1). Eur J Cancer 2009;45:228-47.

14. National Institutes of Health/National Cancer Institute.

Common Terminology Criteria for Adverse Events (CTCAE) Version 4.03; 2009. Available online: http:// www.eortc.be/services/doc/etc/. Accessed June 27, 2014.

15. Therasse P, Arbuck SG, Eisenhauer EA, et al. New guidelines to evaluate the response to treatment in solid tumors. European Organization for Research and Treatment of Cancer, National Cancer Institute of the United States, National Cancer Institute of Canada. J Natl Cancer Inst 2000;92:205-16.

16. Folkman J. Angiogenesis: an organizing principle for drug discovery? Nat Rev Drug Discov 2007;6:273-86.

17. Jubb AM, Oates AJ, Holden S, et al. Predicting benefit from anti-angiogenic agents in malignancy. Nat Rev Cancer 2006;6:626-35.

18. Tsutsumi S, Ishibashi K, Uchida N, et al. Phase II trial of chemotherapy plus bevacizumab as second-line therapy for patients with metastatic colorectal cancer that progressed on bevacizumab with chemotherapy: The Gunma Clinical Oncology group (GCOG) trial 001 SILK study. Oncology 2012;83:151-7.

19. Peeters M, Price TJ, Cervantes A, et al. Randomized phase III study of panitumumab with fluorouracil, leucovorin, and irinotecan (FOLFIRI) compared with FOLFIRI alone as second-line treatment in patients with metastatic colorectal cancer. J Clin Oncol 2010;28:4706-13.

20. Vincenzi B, Santini D, Russo A, et al. Bevacizumab in association with de Gramont 5-fluorouracil/folinic acid in patients with oxliplatin-, irinotecan-, and cetuximabrefractory colorectal cancer: a single-center phase 2 trial. Cancer 2009;115:4849-56.

21. Ziras N, Potamianou A, Varthalitis I, et al. Multicenter phase II study of gemcitabine and oxaliplatin (GEMOX) as second-line chemotherapy in colorectal cancer patients pretreated with 5-fluorouracil plus irinotecan. Oncology 2006;70:106-14.

22. Chang AY, Lopes G, Hsin KW, et al. Phase II trial of 5-fluorouracil/leucovorin/gemcitabine/cisplatin as secondline treatment in patients with metastatic or recurrent colorectal carcinoma: a cancer therapeutics research group study. Clin Colorectal Cancer 2007;6:646-51.

23. Ryanne Wu R, Lindenberg PA, Slack R, et al. Evaluation 
of hypertension as a marker of bevacizumab efficacy. J

Gastrointest Cancer 2009;40:101-8.

24. Langer C, Kopit J, Awad M, et al. Analysis of K-Ras mutations in patients with metastatic colorectal cancer receiving cetuximab in combination with irinotecan: results from the EPIC trial. Ann Oncol 2008;19:viii125-52.

25. Bufill JA. Colorectal cancer: evidence for distinct genetic categories based on proximal or distal tumor location. Ann Intern Med 1990;113:779-88.

26. Missiaglia E, Jacobs B, D'Ario G, et al. Distal and proximal colon cancers differ in terms of molecular, pathological, and clinical features. Ann Oncol 2014;25:1995-2001.

27. Guinney J, Dienstmann R, Wang X, et al. The consensus

Cite this article as: Tsai HL, Huang CW, Ma CJ, Su WC, Chang TK, Chen PJ, Yeh YS, Wang JY. An observational study of vascular endothelial growth factor inhibitors as secondline treatment for metastatic colorectal cancer treated with bevacizumab plus FOLFIRI beyond progression: the association with $R A S$ mutation and tumor sidedness. Transl Cancer Res 2019;8(6):2357-2370. doi: 10.21037/tcr.2019.09.59 molecular subtypes of colorectal cancer. Nat Med 2015;21:1350-6.

28. Ulivi P, Scarpi E, Chiadini E, et al. Right- vs. LeftSided Metastatic Colorectal Cancer: Differences in Tumor Biology and Bevacizumab Efficacy. Int J Mol Sci 2017;18:E1240.

29. Boeckx N, Koukakis R, Op de Beeck K, et al. Effect of Primary Tumor Location on Second- or Later-line Treatment Outcomes in Patients With RAS Wild-type Metastatic Colorectal Cancer and All Treatment Lines in Patients With RAS Mutations in Four Randomized Panitumumab Studies. Clin Colorectal Cancer 2018;17:170-178.e3. 